\title{
Self-Reported Adverse Reactions Among Patients Initiating Antiretroviral Therapy in Brazil
}

Cristiane A. Menezes de Pádua ${ }^{1}$, Cibele C. César ${ }^{2}$, Palmira F. Bonolo ${ }^{1}$, Francisco A. Acurcio ${ }^{3}$ and Mark Drew C. Guimarães ${ }^{1}$

${ }^{1}$ Department of Preventive and Social Medicine, Medicine College, Federal University of Minas Gerais (UFMG); ${ }^{2}$ Department of Statistics, Institute of Exact Sciences - UFMG; ${ }^{3}$ Department of Social Pharmacy, Pharmacy College - UFMG.; Belo Horizonte, MG, Brazil

\begin{abstract}
A cross-sectional analysis was carried out to describe adverse reactions to antiretroviral therapy (ART) reported by HIV-infected patients initiating treatment at two public health AIDS referral centers in Belo Horizonte, Brazil, 2001-2003 and to verify their association with selected variables. Adverse reactions were obtained through interview at the first follow-up visit (first month) after the antiretroviral prescription. Socio-demographic and behavioral variables related to ART were obtained from baseline and follow-up interviews and clinical variables from medical charts. Patients with four or more reactions were compared to those with less than four. Odds ratio with $95 \%$ confidence interval were estimated using logistic regression model for both univariate and multivariate analyses. At least one adverse reaction was reported by $92.2 \%$ of the participants while $56.2 \%$ reported four or more different reactions. Antiretroviral regimens including indinavir/ritonavir, irregular use of antiretrovirals and switch in regimens were independently associated with four or more adverse reactions ( $O R=7.92,5.73$ and 2.03, respectively). The initial period of ARV treatment is crucial and patients' perception of adverse reactions should be carefully taken into account. Strategies for monitoring and management of adverse reactions including the choice of regimens and the prevention of irregular ART should be developed in AIDS/HIV referral centers in Brazil to promote better adherence to antiretroviral therapy.
\end{abstract}

Key-Words: HIV, antiretroviral therapy, adverse reactions, self-report.

Antiretroviral therapy (ART) has markedly changed the pattern of infection by the human immunodeficiency virus (HIV) and the acquired immunodeficiency syndrome (AIDS). Current ART regimens are capable of reducing viral load to undetectable levels with a consequent increase in lymphocyte T-CD4+ counts and a substantial reduction in HIV-associated morbidity and mortality [1]. In spite of ART benefits, adverse reactions to these drugs have been pointed to as one of the main reasons for discontinuation and nonadherence to ART [2-10].

The occurrence of adverse reactions has been described as being high, especially at the beginning of ART, when patients not only recognize them, but also ascribe these undesirable effects to antiretroviral use [4,9,11]. Self-reported adverse reactions by HIV-infected patients have been employed by several authors. They have usually been defined as single symptoms (e.g. nausea, diarrhea), according to their intensity, as well as the report of their absolute number $[12,13]$. Additionally, female [12,14,15] patients given ritonavir compared to other protease inhibitors (PI) [12,14], older patients, hemophiliacs, individuals with hepatitis [14] and immunosuppressed patients receiving nucleoside reverse transcriptase inhibitor (NRTI) [13], have all been identified as being at increased risk for adverse reactions to ART.

Received on 8 June 2006; revised 21 December 2006.

Address for correspondence: Dr. Mark Drew Crosland Guimarães. Department of Preventive and Social Medicine, Medicine College, Federal University of Minas Gerais. Av. Prof. Alfredo Balena $190,10^{\circ}$ andar, Belo Horizonte, MG, Brazil. Zip code: 30.130-100. Phone: + 55313248 9103; Fax: + 55313248 9109. E-mail: drew@medicina.ufmg.br. Financial support: PAHO, PN-DST/AIDS, Brazil, UNESCO.

The Brazilian Journal of Infectious Diseases 2007;11(1):20-26. (C) 2007 by The Brazilian Journal of Infectious Diseases and Contexto Publishing. All rights reserved.
More recently, results of a prospective study on adherence to initial ART carried out by our group [2] have demonstrated that patients reporting higher number of adverse reactions to ART are more likely to be non-adherent to their ART regimens. This indicates that adverse reactions can interfere with the everyday activities of patients, thereby leading to interruption of treatment as well as switches in the prescribed regimens.

To our knowledge, there has been no published data regarding self-reported adverse reactions to antiretrovirals at the beginning of therapy in Brazil, where ART is universally available at public AIDS referral centers. Thus, our objective was to further explore the occurrence of adverse reactions to ART in the first month of treatment, self-reported by HIVinfected patients. In addition, we assessed whether sociodemographic, behavioral, clinical and variables related to ART use were associated to number of different types of adverse reactions.

\section{Materials and Methods \\ Population}

This study included participants of a concurrent prospective study on adherence to ART carried out in two AIDS/HIV public AIDS referral centers in Belo Horizonte, Brazil: Orestes Diniz Training and Reference Center (CTR) from Belo Horizonte City Health Department/Federal University of Minas Gerais and Eduardo de Menezes Hospital (HEM) from Minas Gerais State Health Foundation, from 2001 to 2003 [2]. Briefly, recruitment criteria were documented HIV positive status, at least 18 years of age and to have never been treated with ART. Participation in the study was voluntary and written informed consent was obtained from all patients. The project was submitted and approved by the Ethical Research Committee of the Federal University of Minas Gerais (ETIC 106/99). 
Participants were interviewed at baseline and followed-up for up to ten months following their first ART prescription. Standardized interviews were performed at baseline and at the $1^{\text {st }}, 4^{\text {th }}$ and $7^{\text {th }}$ month of follow-up while medical records were reviewed for all patients in the first year of treatment with these drugs. For the current analysis we only considered the first follow-up visit.

\section{Definition of Outcome and Exposure Variables \\ Adverse Reactions}

In this analysis, adverse reaction refers to any effect or undesirable symptom reported by the patients at the first follow-up interview, perceived as potentially resulted from ART use. During the interview, a structured and standardized questionnaire was used to collect information on gastrointestinal (change in taste, diarrhea, heartburn/stomach pain, nausea, vomiting, sore month), neurological (dizziness, hallucination, headache, insomnia, nightmares), dermatological (allergy), anemia, fatigue, fever and other freely reported reactions. Patients were specifically asked if they had experienced each one of these. This way, the outcome of interest was the number of different types of adverse reactions which had occurred at least once since they had initiated ART. To determine their degree of severity, patients answered for the occurrence of switches in the ART regime due to adverse reactions. To supplement this information, medical charts were examined for cases of hospitalization or death associated with adverse reactions.

\section{Exposure Variables}

Socio-demographic (age, gender, race, marital status, schooling, individual income in the last month and in the prior six months before the interview, and health insurance), and behavioral variables (alcohol use, ever use of illicit drugs and current cigarette smoking) were obtained at baseline interview. Variables regarding ART (regimen, switch and irregular use) were collected in the first follow-up interview whereas clinical variables (clinical initial staging, initial lymphocyte T-CD4+ count, viral load, AIDS-related and non-AIDS-related diagnoses registered before the first ART prescription, hospitalization between the first ART prescription and the first follow-up interview and possible source of HIV infection) were obtained from medical charts. Irregular ART use was defined as at least one day without taking a specific antiretroviral or one missed daily dose and concomitant use of drugs other than antiretrovirals while clinical classification was assessed according to CDC, 1992 [16].

\section{Statistical Analysis}

Descriptive analysis of participants and adverse reactions were carried out. Overall occurrence of adverse reactions was considered as prevalent cases and was defined as the number of patients who reported at least one type of adverse reaction between baseline and first follow-up interview divided by number of patients who returned for the first follow-up interview.
Logistic regression was employed for both univariate and multivariate analyses. Median number of different types of adverse reactions was considered as the cut-off point. Patients who reported four or more types of reactions were compared to those who reported less than four. The strength of the associations between adverse reactions and selected exploratory variables was estimated by the odds ratio (OR) with 95\% confidence interval. The independent effect of selected variables on adverse reactions was assessed by logistic multivariate analysis. Variables included in the initial model consisted of those statistically associated with adverse reactions to ART in the univariate analysis $(\mathrm{p}<0.20)$ and those with clinical and/or epidemiological relevance. Modeling started with all variables followed by sequential deletion to assess the statistical significance of each one, remaining in the final model only those with a p value of less than 0.05 . Likelihood ratio test was used to compare models and the goodness-of-fit of was assessed by Hosmer-Lemeshow test [17]. In view of the large number of missing data, viral load and lymphocyte T-CD4+ were assessed as dummy variables.

\section{Results}

Descriptive Analysis

Among 406 patients enrolled in the study from 2001 to 2003, 361 (88.9\%) returned for the first follow-up interview when adverse reaction data were collected. There were no statistically significant differences concerning sociodemographic variables and ART regimen prescribed between those participants and those lost to follow-up ( $\mathrm{p}>0.05)$.

The time between baseline and the first follow-up interview ranged from 12 to 248 days, with an average of 43 days (median=29 days). At least one different type of adverse reaction was reported by 333 (92.2\%) patients, while 203 (56.2\%) reported four or more different types of adverse reactions. The average number of adverse reactions did not differ between patients who returned for the 1st follow-up interview prior to or after 60 days following baseline interview (Student's test $\mathrm{t}=1.04 ; \mathrm{p}=0.3$ ). In addition, only three patients returned after eight months. Likewise, the pattern of adverse reactions observed did not differ between these patients.

Table 1 presents the pattern of adverse reactions reported by patients. In general, gastrointestinal events were reported more often, nausea being one of the most frequent adverse reaction, which was also common to all ART regimens. More drug-specific adverse reactions comprised those of the central nervous system (e.g. insomnia, nightmares and dizziness) associated with regimens including efavirenz (EFZ). These events are known to occur during the first few days to weeks after initiating treatment [11].

Socio-demographic and behavioral variables indicated that $41.8 \%$ of the participants were over 35 years old, $45.2 \%$ were females, $60.9 \%$ were single, $65.5 \%$ had $\leq 8$ years of schooling, only $22.6 \%$ had health insurance and $63.4 \%$ had individual income $\leq 1$ minimum wage in the last month (US\$ 80.00). This is similar to Brazilian AIDS cases reported to the Ministry of 
Table 1. Distribution of adverse reactions reported by HIV-infected patients after initiation of antiretroviral therapy according to the total number of events and regimens prescribed

\begin{tabular}{|c|c|c|c|c|c|c|}
\hline \multirow[b]{2}{*}{ Adverse reactions } & \multirow[b]{2}{*}{$\begin{array}{l}N(\%)^{*} \\
(\mathbf{n}=361)\end{array}$} & \multicolumn{2}{|c|}{ Antiretroviral regimen ${ }^{* *}$} & \multirow[b]{2}{*}{$\begin{array}{c}\text { EFZ } \\
(\mathrm{n}=105)\end{array}$} & \multirow[b]{2}{*}{$\begin{array}{c}\text { DV, IDV/RTV,LPV/RTV } \\
\text { I }(\mathrm{n}=42)\end{array}$} & \multirow[b]{2}{*}{$\begin{array}{c}\text { NFV } \\
(n=123)\end{array}$} \\
\hline & & $\begin{array}{c}\text { Mono/Dual } \\
(\mathbf{n}=28)\end{array}$ & $\begin{array}{c}\text { NVP } \\
(n=63)\end{array}$ & & & \\
\hline Allergy & 18.3 & 3.6 & 15.9 & 24.8 & 26.2 & 14.6 \\
\hline Anemia & 8.9 & 14.3 & 7.9 & 3.8 & 4.8 & 13.8 \\
\hline Change in taste & 32.7 & 14.3 & 30.2 & 29.5 & 52.4 & 34.2 \\
\hline Diarrhea & 33.2 & 7.1 & 11.1 & 15.2 & 35.7 & 65.0 \\
\hline Dizziness & 22.2 & 14.3 & 22.2 & 30.5 & 21.4 & 17.1 \\
\hline Fatigue & 36.3 & 35.7 & 39.7 & 29.5 & 38.1 & 39.0 \\
\hline Fever & 12.5 & 3.6 & 9.5 & 16.2 & 14.3 & 12.2 \\
\hline Hallucination & 11.9 & 7.1 & 7.9 & 21.9 & 9.5 & 7.3 \\
\hline Headache & 33.2 & 17.9 & 34.9 & 29.5 & 42.9 & 34.2 \\
\hline $\begin{array}{l}\text { Heartburn/ } \\
\text { stomach pain }\end{array}$ & 39.1 & 32.1 & 44.4 & 33.3 & 54.8 & 37.4 \\
\hline Insomnia & 32.1 & 21.4 & 22.2 & 42.7 & 35.7 & 29.3 \\
\hline Nausea & 51.2 & 32.1 & 63.5 & 43.8 & 59.5 & 52.9 \\
\hline Nightmares & 22.2 & 14.3 & 9.5 & 41.0 & 16.7 & 16.3 \\
\hline Sore mouth & 16.1 & 3.6 & 15.9 & 16.2 & 16.7 & 18.7 \\
\hline Vomiting & 36.0 & 21.4 & 42.9 & 23.8 & 54.8 & 39.8 \\
\hline Other & 31.9 & 10.7 & 20.6 & 26.7 & 33.3 & 18.7 \\
\hline
\end{tabular}

*Frequencies refer to the total number of patients $(n=361)$. Most common adverse reactions are highlighted in bold type for each group. ${ }^{* *}$ Frequencies refer to regimens which included: Mono/dual: AZT=zidovudine/ AZT combinations with ddI=didnosine or 3TC=lamivudine; NVP=nevirapine; EFZ=efavirenz; IDV=indinavir, IDV/RTV=ritonavir or RTV/LPV=lopinavir combinations; NFV=nelfinavir.

Table 2. Distribution of socio-demographic, behavioral and clinical variables, Belo Horizonte - Brazil, 2001-2003 (n=361)

\begin{tabular}{|c|c|}
\hline Variables & $N(\%)^{1}$ \\
\hline \multicolumn{2}{|l|}{ Socio-demographic } \\
\hline Age (> 35 years) & 151 (41.8) \\
\hline Gender (female) & $163(45.2)$ \\
\hline Marital status (single) & $220(60.9)$ \\
\hline Schooling ( $\leq 8$ years) & $235(65.5)$ \\
\hline Individual income ( $\leq 1 \mathrm{MW}$, last month $)^{2}$ & $227(63.4)$ \\
\hline Health insurance & 78 (21.6) \\
\hline Race (non-white) & $267(79.9)$ \\
\hline \multicolumn{2}{|l|}{ Behavioral } \\
\hline Alcohol (ever use) & $307(88.2)$ \\
\hline Illicit drugs (ever use) & $91(26.1)$ \\
\hline Current smoking & 119 (34.2) \\
\hline \multicolumn{2}{|l|}{ Source of infection } \\
\hline Heterosexual & $262(72.6)$ \\
\hline $\mathrm{MSM}^{3}$ & 64 (17.7) \\
\hline Transfusion & $18(5.0)$ \\
\hline Injecting drug use & $12(3.3)$ \\
\hline Other/missing & $5(1.4)$ \\
\hline \multicolumn{2}{|l|}{ Clinical/ART } \\
\hline ART switch & $41(11.4)$ \\
\hline \multicolumn{2}{|l|}{ According to ART regimens ${ }^{4}$} \\
\hline Mono/Dual & $12(29.3)$ \\
\hline NVP & $5(12.2)$ \\
\hline EFZ & $6(14.6)$ \\
\hline
\end{tabular}

Table 2. (continued)

\begin{tabular}{|c|c|}
\hline IDV,IDV/RTV,LPV/RTV & $7(17.1)$ \\
\hline NFV & $11(26.8)$ \\
\hline Irregular ART use & $161(44.6)$ \\
\hline \multicolumn{2}{|l|}{ According to ART regimens ${ }^{5}$} \\
\hline Mono/Dual & $16(9.9)$ \\
\hline NVP & $25(15.5)$ \\
\hline EFZ & $50(31.0)$ \\
\hline IDV,IDV/RTV,LPV/RTV & $22(13.7)$ \\
\hline NFV & $48(29.8)$ \\
\hline Concomitant use of other drugs & 192 (53.3) \\
\hline Initial clinical staging ${ }^{6}(\mathrm{~B} / \mathrm{C})$ & $174(50.4)$ \\
\hline \multicolumn{2}{|c|}{ CD4+ lymphocytes count (cells/mm³) } \\
\hline$>500$ & $28(9.1)$ \\
\hline $200-500$ & $113(36.6)$ \\
\hline$<200$ & $168(54.4)$ \\
\hline \multicolumn{2}{|l|}{ Viral load (copies/mL) } \\
\hline$>85,000$ & $90(25.3)$ \\
\hline AIDS-related diagnoses ${ }^{7}$ & 71 (20.5) \\
\hline Non-AIDS-related diagnoses ${ }^{7}$ & $116(33.4)$ \\
\hline Hospitalization $^{8}$ & 36 (10.3) \\
\hline
\end{tabular}

${ }^{1}$ Missing values were excluded. ${ }^{2} \mathrm{MW}$ : minimum wage=US\$ 80.00. ${ }^{3} \mathrm{Men}$ who had sex with other men. ${ }^{4}$ In relation to the total number of switches $(n=41) .{ }^{5}$ In relation to the total number of irregular ART use reporting $(\mathrm{n}=161) .{ }^{6}$ According to CDC Classification System, 1992: category $\mathrm{A}=$ asymptomatic HIV infection, persistent generalized lymphadenopathy or acute HIV infection, $\mathrm{B}=$ symptomatic, not $\mathrm{A}$ or $\mathrm{C}$ conditions; $\mathrm{C}=\mathrm{AIDS}$ indicator. ${ }^{7}$ Before the first ART prescription. ${ }^{8}$ Between the first ART prescription and the first follow-up interview. 
Health [18]. In addition, most participants were non-white, reported alcohol use and had heterosexual contact as a possible source of HIV exposure (Table 2).

All prescriptions followed Brazilian National Guidelines with twenty-two different initial ART regimens. Mono or dual therapy with zidovudine (AZT) was used by 28 (7.8\%) patients, mostly for prophylaxis of vertical transmission. Triple regimens containing two nucleoside reverse transcriptase inhibitors (NRTI) plus one non-nucleoside reverse transcriptase inhibitor (NNRTI) $(\mathrm{n}=168)$ or two NRTIs plus one protease inhibitor (PI) ( $\mathrm{n}=148)$ were prescribed to $87.5 \%$ of the patients, whereas only 17 (4.7\%) received quadruple therapy, including two NRTIs plus two IPs. Combinations of AZT and lamivudine (3TC) were the most commonly prescribed and the main regimen combinations were AZT + 3TC + efavirenz (EFZ) (25.2\%); AZT + 3TC + nelfinavir (NFV) (24.1\%) and AZT + 3TC + nevirapine (NVP) (14.4\%). Equivalent proportions of patients (53.3\%) concomitantly used drugs other than antiretrovirals. Approximately, half of the participants were symptomatic or had AIDS-indicator conditions and had lymphocyte counts $<200$ cells $/ \mathrm{mm}^{3}$, whereas $25.3 \%$ had viral load $>85,000$ copies $/ \mathrm{mL}$.

Of those patients who had their ART regimen switched $(n=41)$, 23 (56.1\%) ascribed the change in regimen to the occurrence of adverse reactions to ART, whereas $23.0 \%$ of those reporting irregular ART use (i.e. at least one day without taking a specific antiretroviral or one missed daily dose) associated this with adverse reactions. Although ART switching and irregular ART occurred more frequently with some ART regimens, statistically significant differences could not be detected.

The pattern of adverse reactions reported by patients who switched or used their ART regimens irregularly was very similar. However, patients were not always able to ascribe the switches or irregular ART use to a specific adverse reaction, but to attribute them to a pool of events, which they had experienced after initiating treatment. Approximately $10 \%$ of patients had at least one hospital admission during the period, however, none were associated with adverse reactions. Similarly, no adverse reaction leading to death was found in the medical records. This indicates that only mild to moderate adverse reactions during the initial treatment with antiretrovirals were experienced by this population.

\section{Univariate and Multivariate Analyses}

Tables 3 and 4 present the results of the univariate and multivariate analyses, respectively. Single patients, those without hospitalization, with lower CD4+ cell count, with more complex ART regimens as well as those with ART switch or irregular ART use presented a higher proportion of four or more different types of adverse reactions $(p<0.20)$ in the univariate analysis. However, only ART regimens, switch in regimens or irregular ART use were independently associated with four or more adverse reactions $(p<0.05)$. It should be noted the doseresponse trend shown for type of regimens with a higher risk for those with more complex ones (IDV, IDV/RTV, LPV/RTV containing regimens). Finally, goodness-of-fit of the final model was satisfactory (Hosmer-Lemeshow test $=4.73$; $\mathrm{p}=0.58$ ) [17].

\section{Discussion}

Our analysis indicated a high proportion of patients reporting at least one (92.2\%) or four or more different types of adverse reaction (56.2\%) in the initial period of ART. Consistent with other publications, most reactions were acute and typical symptoms due to initial treatment with these drugs while the most frequently reported adverse reactions consisted of gastrointestinal effects $[4,8]$. Generally, these effects cause great discomfort, and therefore can be easily perceived and reported by patients given ART. In addition, it should be noted that all reported reactions were mild to moderate.

Few observational studies have investigated the association between socio-demographic variables and adverse reactions to ART. Indeed, in our analysis adverse reactions were not independently associated with any of these variables. Similarly, clinical variables did not seem to influence the adverse reactions to the initial ART, with exception of hospitalization, which did not remain in the multivariate model. There was an increased proportion of reported regimen switch as a result of adverse reactions to ART. In fact, as patients were on initial ART, switches were more likely to occur due to adverse reactions than therapeutic failure. In addition, the frequency of irregular ART use was considerable, with $23 \%$ of the patients attributing it to adverse reactions. The patients' perception of adverse reactions can potentially contribute to non-adherence and discontinuation of the treatment. As noted by other authors [18,19], the difficulty of HIV-infected patients in adhering to ART or other drugs may be related to adverse reactions, often causing treatment to be interrupted by medical recommendation or patient's decision. These findings confirm the results from our previous publication which indicated adverse reactions as one of the key elements contributing for non-adherence [2].

As expected, the frequency of adverse reactions was different according to the given ART regimen. At the time these regimens were prescribed, they were considered preferable for the treatment of HIV-positive adults and adolescents, according to Brazilian National AIDS Program Guidelines [20]. For that reason, a greater proportion of regimens including associations of AZT/3TC, EFZ, NFV and NVP was observed.

Potential limitations inherent to the study design are loss to follow-up and different times of observation between patients' baseline visit and their $1^{\text {st }}$ follow-up visit. As indicated, no statistically significant difference was observed between participant and those lost to follow-up regarding selected variables, and therefore it should not have interfered with the interpretation of our findings. Different time spans may have influenced the number of adverse reactions reported. We have assessed this by checking the pattern of adverse reactions experiencing by patients with longer period of return. Indeed, 
Table 3. Univariate analysis for comparison of selected variables and adverse reactions to ART, Belo Horizonte - Brazil, 2001-2003

\begin{tabular}{|c|c|c|c|c|}
\hline Variables & $\begin{array}{c}\text { Total } \\
\mathbf{N}^{1}\end{array}$ & $\begin{array}{l}\text { Adverse reactions } \\
\qquad(\geq 4)^{2}\end{array}$ & OR $(95 \% \mathrm{CI})^{3}$ & p-value \\
\hline \multicolumn{5}{|l|}{ Socio-demographic } \\
\hline \multicolumn{5}{|l|}{ Age } \\
\hline$>35$ years old & 151 & $83(55.0)$ & 1.0 & \multirow[t]{2}{*}{0.68} \\
\hline$\leq 35$ years old & 210 & $120(57.1)$ & $0.92(0.60-1.40)$ & \\
\hline \multicolumn{5}{|l|}{ Gender } \\
\hline Male & 198 & $110(55.6)$ & 1.0 & \multirow[t]{2}{*}{0.77} \\
\hline Female & 163 & $93(57.1)$ & $1.06(0.70-1.61)$ & \\
\hline \multicolumn{5}{|l|}{ Race } \\
\hline Non-white & 267 & 152 (56.9) & 1.0 & \multirow[t]{2}{*}{0.76} \\
\hline White & 80 & $44(55.0)$ & $1.08(0.65-1.79)$ & \\
\hline \multicolumn{5}{|l|}{ Marital status } \\
\hline Non-single & 220 & $130(59.1)$ & 1.0 & \multirow[t]{2}{*}{$0.17 *$} \\
\hline Single & 141 & 73 (51.8) & $1.34(0.88-2.06)$ & \\
\hline \multicolumn{5}{|l|}{ Schooling } \\
\hline$\leq 8$ years & 235 & $132(56.2)$ & 1.0 & \multirow[t]{2}{*}{0.96} \\
\hline$>8$ years & 124 & 70 (56.5) & $0.98(0.64-1.53)$ & \\
\hline \multicolumn{5}{|l|}{$\begin{array}{l}\text { Individual income } \\
(\leq 1 \mathrm{MW} \text {, last month) }\end{array}$} \\
\hline Yes & 227 & $127(56.0)$ & 1.0 & \multirow[t]{2}{*}{0.92} \\
\hline No & 131 & 74 (55.5) & $0.98(0.63-1.51)$ & \\
\hline \multicolumn{5}{|l|}{ Health insurance } \\
\hline No & 283 & 157 (55.5) & 1.0 & \multirow[t]{2}{*}{0.58} \\
\hline Yes & 78 & $46(59.0)$ & $0.87(0.52-1.44)$ & \\
\hline \multicolumn{5}{|l|}{ Behavioral } \\
\hline \multicolumn{5}{|l|}{ Current smoking } \\
\hline Yes & 118 & $131(57.0)$ & 1.0 & \multirow[t]{2}{*}{0.86} \\
\hline No & 230 & 66 (55.9) & $0.96(0.61-1.05)$ & \\
\hline \multicolumn{5}{|l|}{ Alcohol (ever use) } \\
\hline No & 41 & $23(56.1)$ & 1.0 & \multirow[t]{2}{*}{0.94} \\
\hline Yes & 307 & 147 (56.7) & $1.02(0.53-1.97)$ & \\
\hline \multicolumn{5}{|l|}{ Illicit drugs (ever use) } \\
\hline No & 257 & $144(56.0)$ & 1.0 & \multirow[t]{2}{*}{0.71} \\
\hline Yes & 91 & $53(58.2)$ & $1.09(0.67-1.78)$ & \\
\hline \multicolumn{5}{|l|}{ Clinical/ART } \\
\hline ART Regimen ${ }^{5}$ & & & & \\
\hline Mono/dual & 28 & 11 (39.3) & 1.0 & \\
\hline NVP & 63 & 32 (50.8) & $1.60(0.65-3.94)$ & 0.31 \\
\hline EFZ & 105 & $60(57.1)$ & $2.06(0.88-4.83)$ & $0.10^{*}$ \\
\hline NFV & 123 & 70 (56.9) & $2.04(0.88-4.72)$ & $0.10^{*}$ \\
\hline IDV,IDV/RTV,LPV/RTV & 42 & 30 (71.4) & $3.86(1.40-10.62)$ & $0.01 *$ \\
\hline Initial clinical staging ${ }^{6}$ & & & & \\
\hline A & 171 & $92(53.8)$ & 1.0 & 0.31 \\
\hline$B / C$ & 174 & $103(59.2)$ & $1.26(0.81-1.91)$ & \\
\hline CD4+ lymphocytes (cells/mı & & & & \\
\hline$>500$ & 28 & $10(35.7)$ & 1.0 & \\
\hline $200-500$ & 113 & $64(56.6)$ & $2.35(1.00-5.54)$ & $0.05^{*}$ \\
\hline$<200$ & 168 & $97(57.7)$ & $2.46(1.07-5.65)$ & $0.03^{*}$ \\
\hline Missing & 52 & $32(61.5)$ & $2.88(1.11-7.48)$ & $0.03^{*}$ \\
\hline
\end{tabular}


Table 3. (continued)

\begin{tabular}{|c|c|c|c|c|}
\hline Variables & $\begin{array}{c}\text { Total } \\
\mathbf{N}^{1}\end{array}$ & $\begin{array}{l}\text { Adverse reactions } \\
\qquad(\geq 4)^{2}\end{array}$ & OR $(95 \% \mathrm{CI})^{3}$ & p-value \\
\hline \multicolumn{5}{|c|}{ Viral load (copies/mL) } \\
\hline$\leq 85,000$ & 148 & $80(54.1)$ & 1.0 & \\
\hline$>85,000$ & 90 & $52(57.8)$ & $1.16(0.69-1.97)$ & 0.57 \\
\hline Missing & 123 & $71(57.7)$ & $1.16(0.72-1.88)$ & 0.54 \\
\hline \multicolumn{5}{|c|}{ AIDS-related diagnoses ${ }^{7}$} \\
\hline No & 276 & $156(56.5)$ & 1.0 & 0.98 \\
\hline Yes & 71 & 40 (56.3) & $0.99(0.59-1.68)$ & \\
\hline \multicolumn{5}{|c|}{ Non-AIDS-related diagnoses ${ }^{7}$} \\
\hline No & 231 & 132 (57.1) & 1.0 & 0.73 \\
\hline Yes & 116 & $64(55.2)$ & $0.92(0.59-1.45)$ & \\
\hline \multicolumn{5}{|c|}{ Hospitalization $^{8}$} \\
\hline Yes & 36 & $24(66.7)$ & 1.0 & $0.18^{*}$ \\
\hline No & 314 & $173(55.1)$ & $1.63(0.79-3.37)$ & \\
\hline \multicolumn{5}{|l|}{ ART switch } \\
\hline Yes & 41 & $33(80.5)$ & 1.0 & $0.00 *$ \\
\hline No & 320 & $170(53.1)$ & $3.64(1.63-8.12)$ & \\
\hline \multicolumn{5}{|c|}{ Irregular ART use } \\
\hline No & 200 & $97(48.5)$ & 1.0 & $0.00 *$ \\
\hline Yes & 161 & 106 (65.8) & $2.04(1.33-3.14)$ & \\
\hline \multicolumn{5}{|c|}{ Concomitant use of other drugs } \\
\hline No & 168 & $89(53.0)$ & 1.0 & 0.26 \\
\hline Yes & 192 & $113(58.9)$ & $1.26(0.84-1.93)$ & \\
\hline
\end{tabular}

${ }^{1}$ Missing values were excluded. ${ }^{2}$ Number and proportion of patients reporting 4 or more adverse reactions. ${ }^{3}$ Odds ratios (OR) were obtained using logistic regression model. ${ }^{4} \mathrm{MW}$ : minimum wage $=\mathrm{US} \$ 80.00 .{ }^{5} \mathrm{ART}$ regimens: Mono/dual: AZT=zidovudine/ AZT combinations with ddI=didnosine or 3TC=lamivudine; $N V P=$ nevirapine; $E F Z=e f a v i r e n z ;$ IDV=indinavir, IDV/RTV=ritonavir or RTV/LPV=lopinavir combinations; NFV=nelfinavir. ${ }^{6}$ According to CDC Classification System, 1992: category A=asymptomatic HIV infection, persistent generalized lymphadenopathy or acute HIV infection, $\mathrm{B}=$ symptomatic, not $\mathrm{A}$ or $\mathrm{C}$ conditions; $\mathrm{C}=\mathrm{AIDS}$-indicator. ${ }^{7}$ Before the first ART prescription. ${ }^{8}$ Between the first ART prescription and the first follow-up interview. *Statistically significant at a p value of less than 0.20 .

Table 4 - Final model of the multivariate analysis obtained for the adverse reactions to ART among HIV-infected patients, Belo Horizonte - Brazil, 2001-2003 (n=361)

\begin{tabular}{lcc}
\hline Variables $^{*}$ & OR $(\mathbf{9 5 \% \mathbf { C I } )}$ & p-value \\
\hline ART regimen & *** & \\
Mono/dual & 1.0 & \\
NVP & $3.65(1.26-10.61)$ & 0.00 \\
EFZ & $4.63(1.67-12.83)$ & 0.02 \\
NFV & $4.74(1.73-13.00)$ & 0.00 \\
IDV,IDV/RTV,LPV/RTV & $7.92(2.47-25.37)$ & 0.00 \\
ART switch (yes) & $5.73(2.16-3.18)$ & 0.00 \\
Irregular ART use (yes) & $2.03(1.30-3.18)$ & 0.00 \\
\hline
\end{tabular}

${ }^{*}$ Risk categories are indicated in parenthesis. ${ }^{* *}$ Odds ratios (OR) were obtained using logistic regression model. ${ }^{* * *}$ ART regimens: Mono/dual: $\mathrm{AZT}=$ zidovudine/AZT combinations with $\mathrm{ddI}=$ didnosine or 3TC=lamivudine; NVP=nevirapine; EFZ=efavirenz; IDV=indinavir, IDV/ $\mathrm{RTV}=$ ritonavir or RTV/LPV=lopinavir combinations; NFV=nelfinavir.

they reported slightly higher number of adverse reactions on average, but the differences were not statistically significant and the degree of severity of their adverse reactions was also similar. Caution should also be considered on the direction of the associations found. Irregular use or switch in regimen are more likely to be consequences of adverse reactions.

Our study assessed adverse reactions occurring within approximately the first 30 days of ART; a period during which inherent symptoms of HIV related to altered immunity may be present. Therefore, adverse reactions reported by patients could be confounded with symptoms due to HIV, not resulting directly from ART. This could potentially lead to an overestimation of this outcome. Nevertheless, although causation of the adverse effects cannot be indisputably determined, any undesired effect perceived by patients as having been due to ART should be taken into account by health professionals. The beginning of therapy is the period during which adverse reactions or undesirable symptoms will likely, to a large extent, contribute to non-adherence to ART. We should also point out that, number of different types of events may be a suitable way of expressing the effect of a set of adverse reactions in the initial therapy, especially in situations when only mild to moderate reactions are observed, as it is our case. Strategies, such as the establishment of standardized protocols for acute and long-term effects of ART should be implemented in public AIDS/HIV referral centers in Brazil, as well as better counseling and management of adverse 
reactions by health professionals. A more precise and accurate assessment of adverse reactions by health professionals can promote better adherence to these drugs, facilitate an appropriate early ART switch in regimen. This examination of adverse effects may also help health professionals choose regimens better suited to the patients, thereby preventing irregular ART use and the need for early switch in regimens.

\section{References}

1. Sepkowitz K.A. AIDS - the first 20 years. N Engl J Med 2001,344:1764-72.

2. Bonolo P.D., Cesar C.C., Acurcio FA, et al. Non-adherence among patients initiating antiretroviral therapy: a challenge for health professionals in Brazil. AIDS 2005;19 Suppl 4:S5-S13.

3. Dieleman J.P., Jambroes M., Gyssens I.C., et al. Determinants of recurrent toxicity-driven switches of highly active antiretroviral therapy. The ATHENA cohort. AIDS 2002;16:737-45.

4. Duran S., Spire B., Raffi F., et al. Self-reported symptoms after initiation of a protease inhibitor in HIV-infected patients and their impact on adherence to HAART. HIV Clinical Trials 2001;2:38-45.

5. Lignani L., Jr., Greco D.B., Carneiro M. Avaliação da aderência aos anti-retrovirais em pacientes com infecção pelo HIV/Aids. Rev Saude Publica 2001;35:495-501.

6. Mocroft A., Youle M., Moore A., et al. Reasons for modification and discontinuation of antiretrovirals: results from a single treatment centre. AIDS 2001;15:185-94.

7. Ammassari A., Murri R., Pezzotti P., et al. Self-reported symptoms and medication side effects influence adherence to highly active antiretroviral therapy in persons with HIV infection. J Acquir Immune Defic Syndr 2001;28:445-9.

8. Bonfanti P., Valsecchi L., Parazzini F., et al. Incidence of adverse reactions in HIV patients treated with protease inhibitors: a cohort study. J Acquir Immune Defic Syndr 2000;23:236-45.
9. d’Arminio Monforte A., Lepri A.C., Rezza G., et al. Insights into the reasons for discontinuation of the first highly active antiretroviral therapy (HAART) regimen in a cohort of antiretroviral naive patients. AIDS 2000;14:499-507.

10. van Roon E.N., Verzijl J.M., Juttmann J.R., et al. Incidence of discontinuation of highly active antiretroviral combination therapy (HAART) and its determinants. J Acquir Immune Defic Syndr Hum Retrovirol 1999;20:290-4.

11. Carr A., Cooper D.A. Adverse effects of antiretroviral therapy. Lancet 2000;356:1423-30.

12. Lucas G.M., Chaisson R.E., Moore R.D. Highly active antiretroviral therapy in a large urban clinic: risk factors for virologic failure and adverse drug reactions. Ann Intern Med 1999;131:81-7.

13. Moore R.D., Fortgang I., Keruly J., Chaisson R.E. Adverse events from drug therapy for human immunodeficiency virus disease. Am J Med 1996;101:34-40.

14. Bonfanti P., Ricci E., Landonio S., et al. Predictors of protease inhibitor-associated adverse events. Biomed Pharmacother 2001; 55:321-3.

15. Currier J.S., Spino C., Grimes J., et al. Differences between women and men in adverse events and CD4+ responses to nucleoside analogue therapy for HIV infection. The Aids Clinical Trials Group 175 Team. J Acquir Immune Defic Syndr 2000;24:316-24.

16. CDC. 1993 Revised classification system for HIV infection and expanded surveillance case definitions for AIDS among adolescents and adults. MMWR 1992;41:RR-17.

17. Collett D. Modeling Survival Data in Medical Research. London: Chapman\& Hall; 1994.

18. Acurcio F.A., César C.C., Guimarães M.D.C. Health care utilization and survival among patients with AIDS in Belo Horizonte, Minas Gerais, Brazil. Cad Saude Publica 1998;14:811-20.

19. Heath K.V., Singer J., O’Shaughnessy M.V., et al. Intentional nonadherence due to adverse symptoms associated with antiretroviral therapy. J Acquir Immune Defic Syndr 2002;31:211-7.

20. Brasil. Recomendações para terapia anti-retroviral em adultos e adolescentes infectados pelo HIV- 2001. http://www.aids.gov.br/ (Accessed October 10, 2003). 